\title{
Bridging the resolution gap: correlative super-resolution imaging
}

\section{lan M. Dobbie}

This month's Under the Lens discusses progress towards bridging the resolution gap in correlative super-resolution light and electron microscopy, and highlights its application for visualizing bacterial infections.

Electron microscopy (EM) gives the best achievable imaging resolution; however, it is often difficult or impossible to locate specific proteins or structures in EM images. A wide range of techniques in fluorescence microscopy enable imaging of specific proteins or structures. Combining these techniques leads to so called correlative light and electron microscopy (CLEM), where the sample is first imaged on a fluorescence microscope and then imaged on an electron microscope ${ }^{1}$. One substantial issue in CLEM studies is the large difference in resolution between the fluorescence images $(250-400 \mathrm{~nm})$ and the EM images $(2-10 \mathrm{~nm})$. An obvious development is to use super-resolution fluorescence imaging to narrow this resolution gap and improve the quality and utility of the correlated images.

The power of CLEM is especially important for locating and examining biological interactions that take place at a small scale and with low frequency. This closely describes important questions in microbiology such as pathogen cell entry, immune escape and host-pathogen interactions that lead to disease. CLEM can provide vital experimental evidence to help answer many of these questions by identifying and locating rare events for visualization in the electron microscope and then unambiguously identifying proteins, or other cellular components, at these loci.

EM sample preparation techniques are harsh and preclude the use of standard fluorescence labelling techniques. Fluorescent proteins are usually dehydrated by the processing, losing most, if not all, of their fluorescence. Antibodies used for immune labelling often provide low labelling efficiencies, especially on embedded samples where access of the antibody to the target sites can be difficult. To overcome these challenges, van Elsland et al. ${ }^{2}$ present an interesting new labelling strategy for CLEM, using non-canonical amino acid analogues to label a population of Salmonella enterica subsp. enterica serovar Typhimurium. S. Typhimurium are fed homopropargylglycine (Hpg; an analogue of methionine), which is then incorporated into bacterial proteins. The labelled bacteria are used to infect cells and the infected cells are then prepared for EM using the Tokuyasu method, a mild processing approach with minimal fixation followed by cryosectioning. The Hpg is then fluorescently labelled using click chemistry. These samples are imaged using fluorescence stochastic optical reconstruction microscopy (STORM), a variant of single molecule localisation microscopy, and finally imaged on a transmission electron microscope.

Using this method, the authors were able to image $S$. Typhimurium proteins within host cells at a resolution of $20 \mathrm{~nm}$, massively improving the resolution by over tenfold. The future application of this method could lead to a more detailed understanding of the intracellular lifestyle of Salmonella spp., for example, molecular interactions that occur when the bacterium escapes from the Salmonella-containing vacuole, enabling pathogen dissemination.

In an unrelated development, Wang et al. ${ }^{3}$ demonstrate a new lens design that enables very high-resolution STORM imaging of bacteria under cryogenic conditions. Light microscopy under cryogenic conditions is complicated by the requirement to insulate the lens at room temperature from the sample at cryogenic temperatures. This requires the use of an air lens with a relatively low

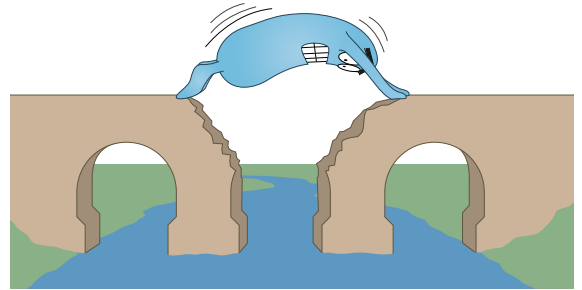

Credit: Philip Patenall/Springer Nature Limited

numerical aperture (a measure of the capacity of a lens to gather light and resolve fine specimen detail), reducing light collection and resolution. The authors used a solid immersion lens to overcome this limitation, achieving a higher numerical aperture. They then used this setup to collect single molecule localization microscopy images of a GFP-tagged putative antibacterial peptide ABC transporter in Escherichia coli, in which cell membranes were stained with a red fluorescent probe, achieving a localization precision of $7 \mathrm{~nm}$ in single colour and $\sim 10 \mathrm{~nm}$ in dual colour imaging.

Altogether, these studies make major strides towards bridging the resolution gap between light microscopy and EM in super-resolution CLEM. I anticipate new developments in the coming years that will further bridge this gap, leading to a clearer picture of host-pathogen interactions.

Ian M. Dobbie

Micron Advanced Bioimaging Unit, Department of Biochemistry, University of Oxford, Oxford, UK. e-mail: underthelens@bioch.ox.ac.uk https://doi.org/10.1038/s41579-019-0203-8

Hampton, C. M. et al. Correlated fluorescence microscopy and cryo-electron tomography of virus-infected or transfected mammalian cells. Nat. Protoc. 12, 150-167 (2017).

2. van Elsland, D. M. et al. Ultrastructural imaging of Salmonella-host interactions using super-resolution correlative light-electron microscopy of bioorthogonal pathogens. ChemBioChem 19, 1766-1770 (2018).

3. Wang, L. et al. Solid immersion microscopy images cells under cryogenic conditions with $12 \mathrm{~nm}$ resolution. Commun. Biol. 2, 74 (2019).

\section{Competing interests}

The author declares no competing interests. 